\title{
Development of extreme narrow band filter for LGS daytime use
}

\author{
Yutaka Hayano (Advanced Technology Center / TMT-Japan Project Office, NAOJ),
}

Mark Chun (Institute for Astronomy, University of Hawaii), Mitsuhiko Honda (Kurume University), Chris Packham (University of Texas)

\section{Introduction}

The use of laser guide star (LGS) in daytime expands the operational time of telescope for $\mathrm{AO}$ instruments, especially for thermal infrared wavelengths, because the sky brightness is not so different from night to daytime. However, the daytime sky background at $589 \mathrm{~nm}$ is about $10^{6}$ times brighter than a bright night. However, as the bandwidth of sodium D2 line is a few $\mathrm{GHz}$, an extremely narrow bandpass filter at this wavelength makes it possible to use LGSs during the day.

Begin with developing an interference filter, we found that the spectral bandwidth could not be achieved at the level of $1 \mathrm{~nm}$. Then we started to investigate etalon technology to achieve an extremely narrow band filter. We focused on two different technologies, one is an air-gap etalon and another is a thin etalon layer sandwiched by the high reflective coating. We present the prototype fabrication of etalons based on both technologies and report the results of measurement of the peak transmission and spectral bandwidth.

\section{Top-level specification of NBP filter}

\begin{tabular}{|c|c|c|}
\hline PARAMETER & VALUE and TOLERANCE & $\begin{array}{c}\text { TOLERANCE } \\
\text { NOTES }\end{array}$ \\
\hline \multicolumn{3}{|l|}{ General } \\
\hline Operating/Working Temperature & $0^{\circ} \mathrm{C} \pm 10^{\circ} \mathrm{C}$ & "Operating Temperature" \\
\hline Central Wavelength & $589.16 \mathrm{~nm}$ & "Operating Wavelength" \\
\hline \multicolumn{3}{|l|}{ Optical Design } \\
\hline Surface shape & plano-plano & \\
\hline Working aperture (all FOV) & $30 \mathrm{~mm}$ & clear aperture \\
\hline Angle of incidence & $0+-1$ deg & \\
\hline \multicolumn{3}{|l|}{ Surface Quality } \\
\hline Surface quality & $60 / 40$ & scratch and dig \\
\hline Transmitted wavefront & 入/10 RMS@632nm & over clear aperture prior to coating \\
\hline \multicolumn{3}{|l|}{ Optical Coating } \\
\hline Central Wavelength & $589.16 \mathrm{~nm}$ & at operating temp \\
\hline Transmitted Bandpass & FWHM $<0.3 \mathrm{~nm}$ & at operating temp \\
\hline Peak transmission & $\mathrm{T}>50 \%$ at $589.16 \mathrm{~nm}$ & \\
\hline Out of bounds transmissivity & $>$ OD6 from 400 to $1100 \mathrm{~nm}$ & at operating temp \\
\hline \multicolumn{3}{|l|}{ Substrate Properties } \\
\hline Substrate Material & - & At discretion of vendor \\
\hline Optic shape & Circular & \\
\hline Optic size & $35.0 \mathrm{~mm}$ diameter $+0.00 /-0.1 \mathrm{~mm}$ & See Note 1 \\
\hline Optic thickness & $<8 \mathrm{~mm} \pm 0.25 \mathrm{~mm}$ & See Note 1 \\
\hline Bevel & $0.3 \mathrm{~mm} \times 45 \mathrm{deg}$ & Standard bevel \\
\hline \multicolumn{3}{|l|}{ Miscellaneous } \\
\hline Quantity & $1-2$ & no spare \\
\hline
\end{tabular}

\section{Air-gapped etalon filter}

The table below shows a parameters of air-gapped etalon. The bandpass range can be achieved $0.1 \mathrm{~nm}$ FWHM, though, transmission at wavelength other than sodium D2 line is higher than our specification. Therefore, we have to add another bandpass filter, whose bandpass width is around 1 or $2 \mathrm{~nm}$. But this is not a critical challenge to achieve.

\begin{tabular}{|l|l|}
\hline Parameter & Value \\
\hline Reflectivity of etalon & $81 \%$ \\
\hline Finesse & 15 \\
\hline Free spectrum range & $1.5 \mathrm{~nm}$ \\
\hline Bandpass width & $0.1 \mathrm{~nm}$ \\
\hline Air gap & 115.3 micron \\
\hline
\end{tabular}

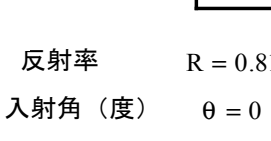

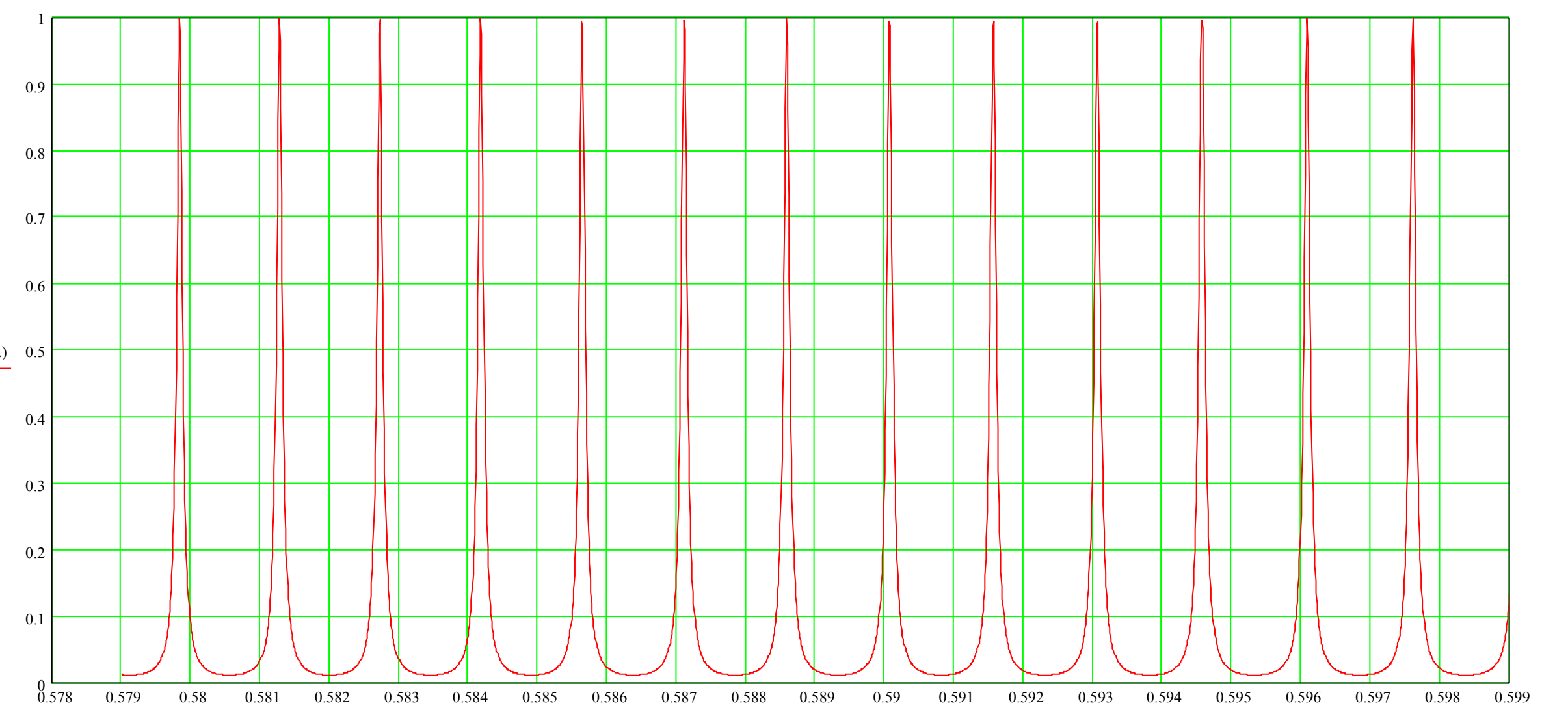

(8)

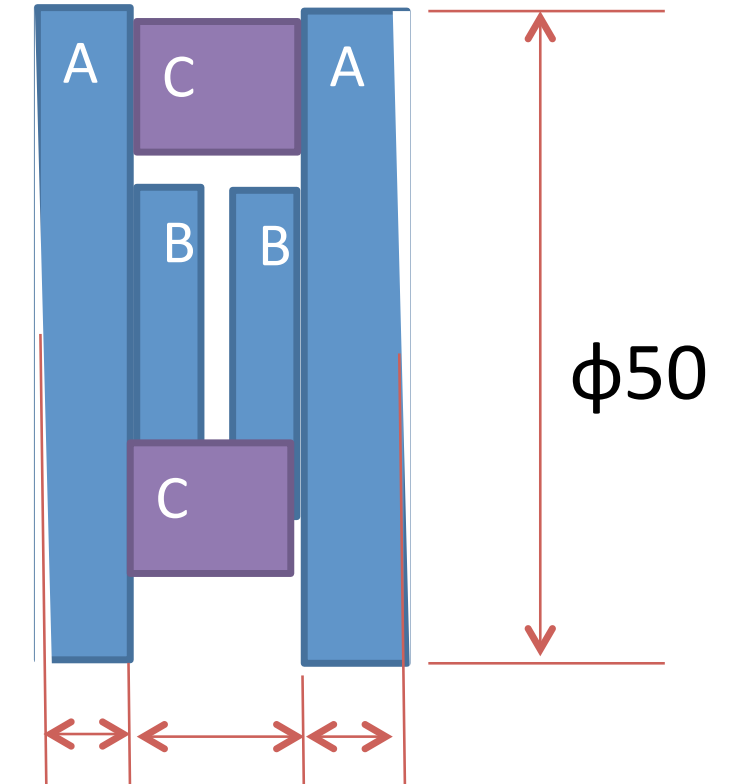

(6) (9) (6)
The second candidate of etalon filter is designed by using multi-layer coating technology. As you see in the figure left below, we develop several types of multi-layers on the substrate, made by fused silica. The layer in the middle is sandwiched by high reflective layers, which act as a etalon. The thickness of middle layer is designed from 1 to 4 micron. The total throughput can be tuned by the reflectivity of HR layers. It is difficult to control the exact thickness of middle layer so that the central wavelength of etalon filter is slightly longer than sodium D2 line. Thus we tune the central wavelength by tilting the etalon unit. According to the model calculation, we can tune about $3 \mathrm{~nm}$ by tilting the etalon by 8 degrees.

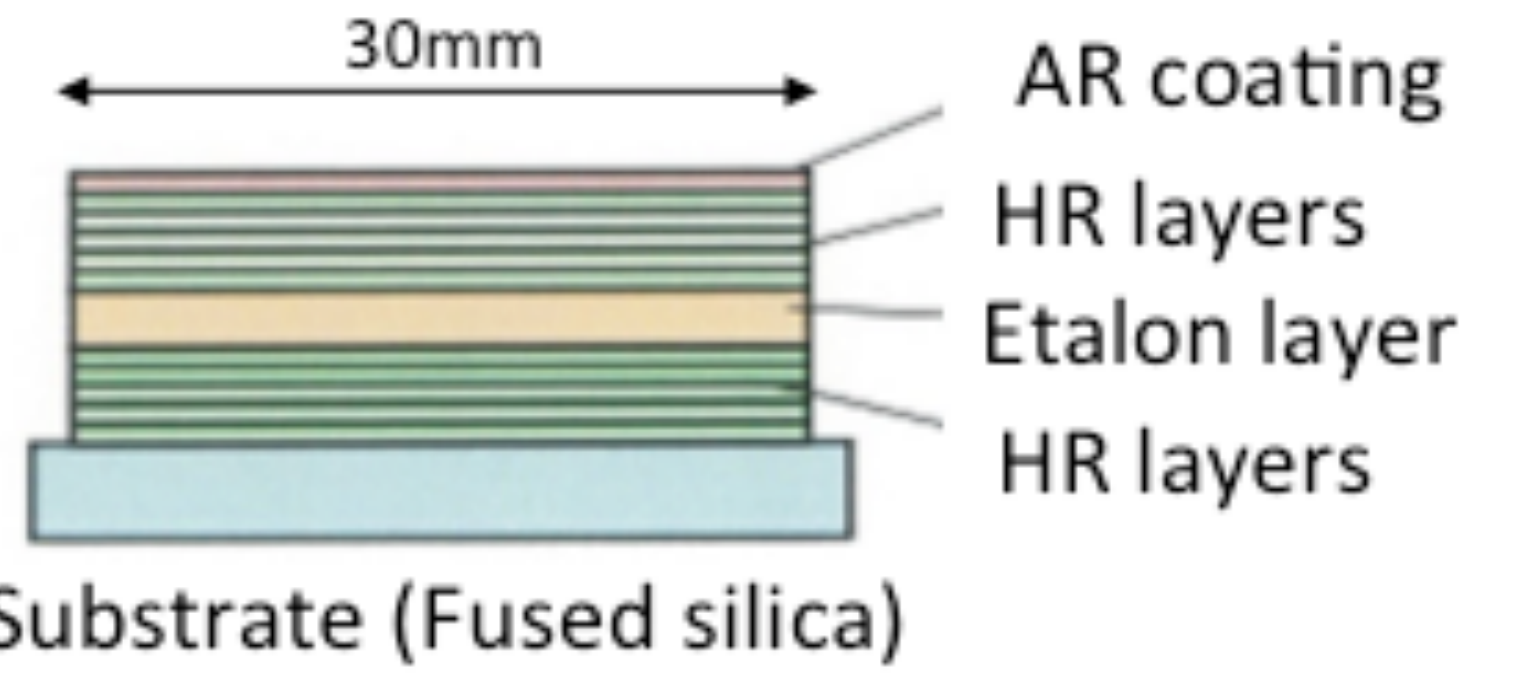

\section{Characteristics of etalon filter}

The experimental setup is shown on the left figure. The laser beam at the wavelength of $589 \mathrm{~nm}$ is fed into the spatial filter and collimated by the lens. etalon filter is mounted on the rotational stage to adjust the incident angle of the laser collimated beam. The peak transmission of air-gap etalon reached around $80 \%$, the spectral bandwidth is approximately $0.1 \mathrm{~nm}$ and the free spectral range (FSR) is about $1.5 \mathrm{~nm}$, which are matched with the design specification. The next challenge of air-gap etalon is how to block the adjacent spectral transmission. For the thin etalons with high-reflective coating, the peak transmission is distributed around $40 \%$ to $70 \%$, the spectral bandwidth is about $0.15 \mathrm{~nm}$ to $0.6 \mathrm{~nm}$. Thus, the designed specification and the measurement results have been not exactly matched, but we found some empirical relationship between these performance parameters. Also the central transmission wavelength varies across the etalon aperture. Finally, we propose the second

prototype fabrication of thin etalon sandwiched by high reflective coating, including the experience and results of first prototype fabrication.

\section{Multi-layered etalon filter}
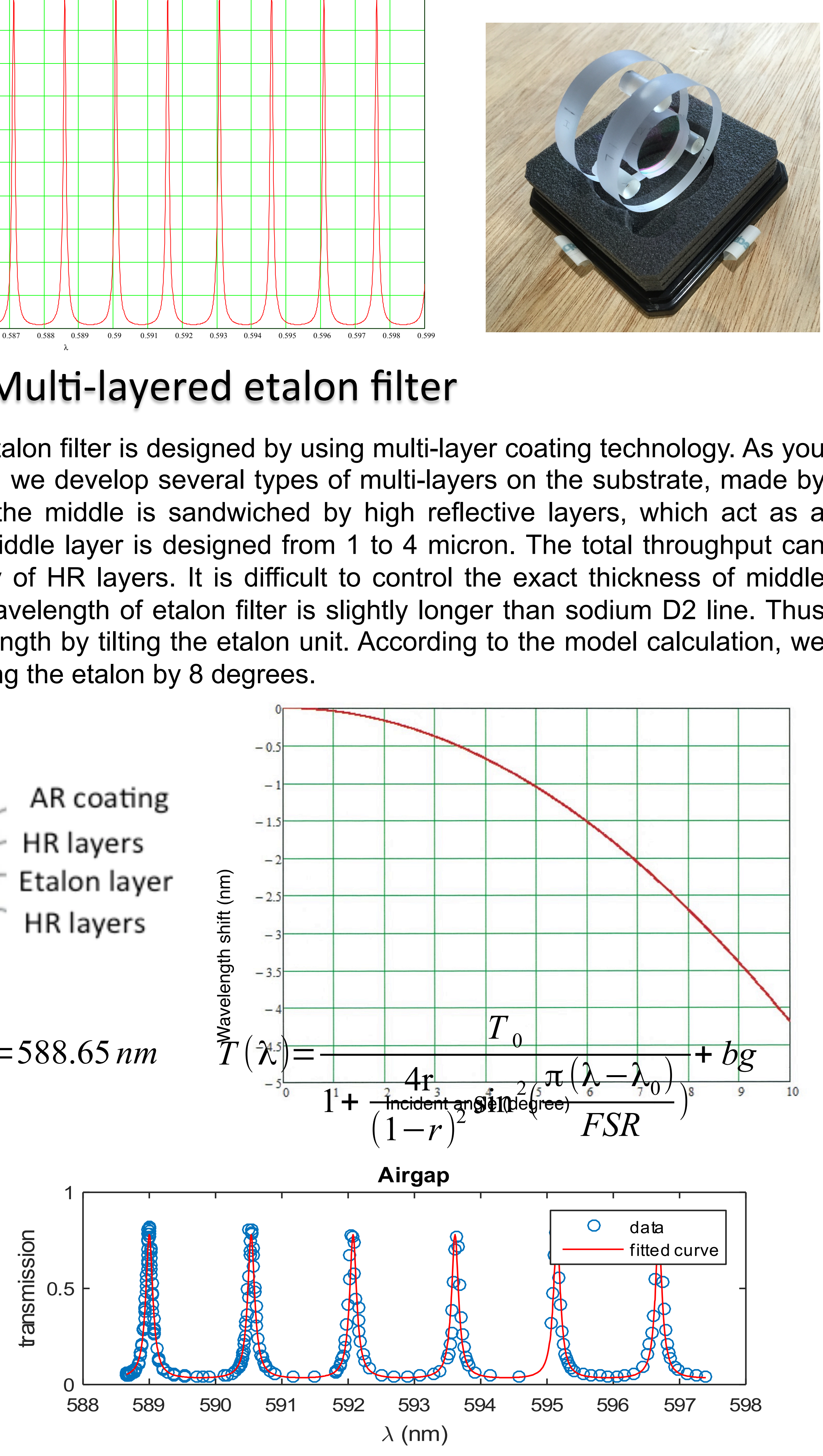

Multi-layered etalon filter

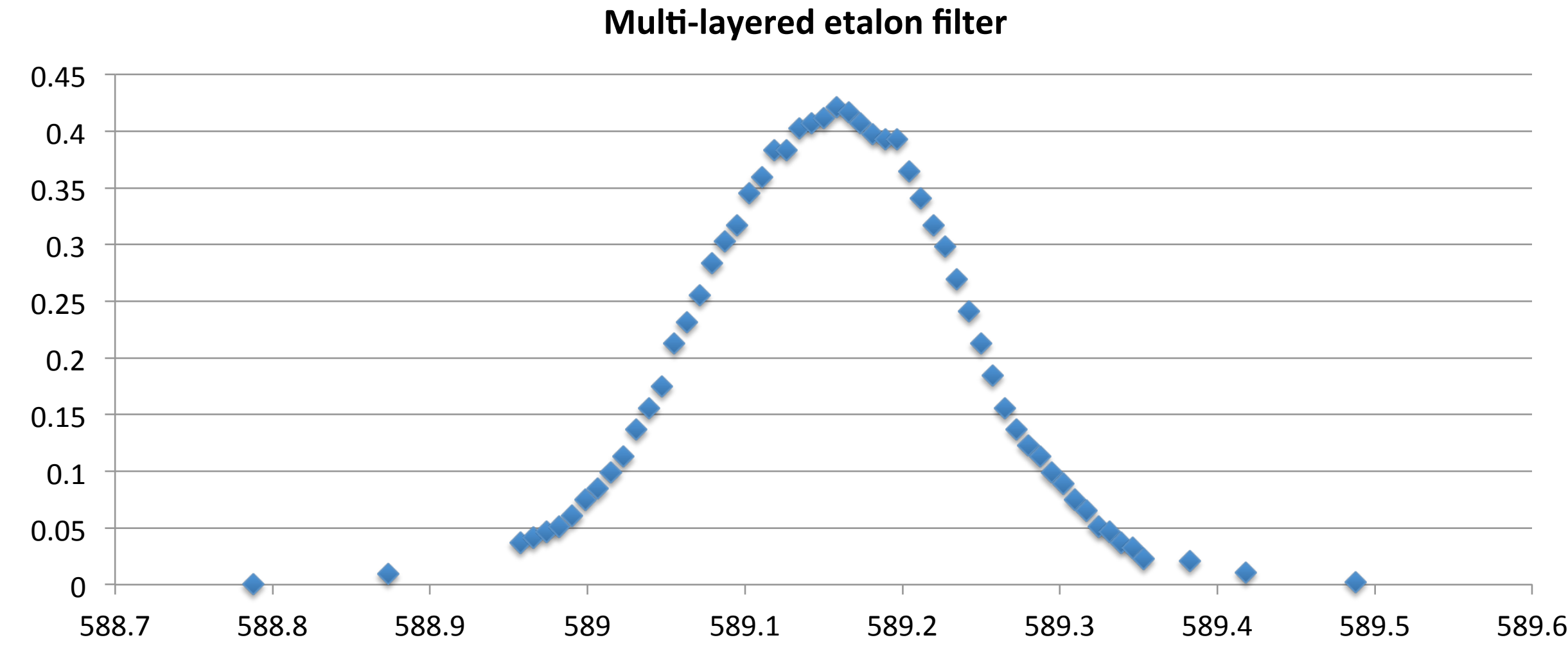

